\title{
Metal-Free Visible-Light-Induced Dithiol-Ene Clicking via Carbon Nitride to Valorize 4-Pentenoic Acid as a Functional Monomer
}

\author{
Baris Kumru, ${ }^{+, \dagger}$ Jose Mendoza Mesa, ${ }^{\ddagger \dagger}$ Markus Antonietti, ${ }^{\dagger}$ and Majd Al-Naji* ${ }^{+\dagger}$ \\ ${ }^{\dagger}$ Max Planck Institute of Colloids and Interfaces, Am Mühlenberg 1, 14476 Potsdam, Germany \\ Supporting Information
}

ABSTRACT: The necessity for more bio-based building blocks and processes have led to utilizing new polymerization approaches with a lower carbon footprint. Here, we demonstrate the synthesis of a visible-light-induced dithiol-ene clicking reaction between lignocellulosic biomass-derivable 4pentenoic acid (4-PEA) and different dithiols, i.e., 1,2-ethanedithiol (EDT), 2,2-(ethylenedioxy)diethanethiol (EDDT), and 1,4-benzenedimethanethiol $(\mathrm{BDT})$, using graphitic carbon nitride $(g-\mathrm{CN})$ as a metal-free photocatalyst. The formation of dicarboxylic acid functional monomers were confirmed using ${ }^{1} \mathrm{H}$ NMR and FT-IR. Furthermore, polyamides were synthesized from the dicarboxylic acid functional monomers to demonstrate the applicability of the monomers yielding new polyamide end polymers.

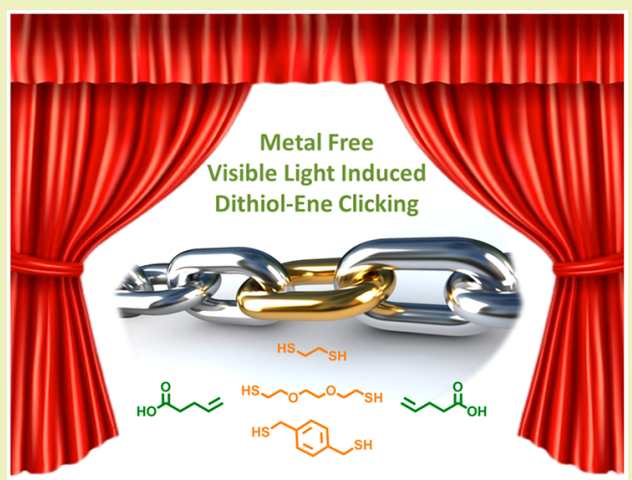

KEYWORDS: Photochemistry, Carbon nitride, Pentenoic acid, Dithiols, Dithiol-ene clicking, Bio-derived polymer

\section{INTRODUCTION}

In order to reduce the dependency on fossil resources, the synthesis of biobased building blocks from renewable resources is of a great interest. ${ }^{1}$ Lignocellulosic biomass, i.e., a mixture of cellulose, hemicellulose, and lignin as found in wood or bagasse, is a promising starting product owing to its low costs, availability, functionality, and reactivity. ${ }^{2}$ In this context, a wide range of building blocks was already derived from lignocellulosic biomass, such as levulinic acid, $\gamma$-valerolactone (GVL), 5hydroxymethylfuran, and 2,5-dimethylfuran. GVL is especially easy to synthesize and already a platform chemical. ${ }^{3}$ In the presence of an acid catalyst, GVL undergoes a ring-opening reaction to pentenoic acid isomers, i.e., 2-, 3-, and 4-pentenoic acid (PEAs), which are relevant for fragrance or the pharmaceutical industry. ${ }^{4-6}$ Additionally, PEAs have been already explored as a starting material for the synthesis of different biobased monomers and polymers, e.g., adipic acid, C6-C8 unsaturated carboxylic acids, amides, and esters. ${ }^{7-10}$ Among PEAs, 4-pentenoic acid (4-PEA) possesses the potential to produce a new biomass-derived dicarboxylic acid via thiol-ene click chemistry. Such dicarboxylic acid monomers can be condensed with diamines for polyamide synthesis. $^{11}$

Click chemistry offers an unique platform for the efficient synthesis of functional small molecules and polymers. ${ }^{12,13}$ Specifically, thiol-ene clicking grants covalent connection of ene-functionalities with thiol groups with practically perfect yields under green conditions. ${ }^{14}$ This chemistry is generally mediated via typical radical thermal initiators such as azobis(isobutyronitrile) (AIBN) ${ }^{15}$ or light-induced reactions by utilizing light-absorbing metal compounds, e.g., $\mathrm{TiO}_{2}$ and
$\left(\mathrm{Ru}(\mathrm{bpz})_{3}{ }^{2+}\right) \cdot{ }^{16,17}$ Very recently, $\mathrm{ZnIn}_{2} \mathrm{~S}_{4}$ was introduced as a visible light photocatalyst for thiol clicking reactions and showed potential for alkyne-thiol addition. However, the yields of alkene-thiol addition were considerably low despite long reaction periods $(15 \mathrm{~h})$ and a small amount of reactant (in mmol range). ${ }^{18}$ The versatility of thiol-ene clicking could be extended to more green conditions via utilization of visible light and metal-free catalysts while targeting high yields in a short reaction time and possible scalability. The discussed alternative, graphitic carbon nitride $(g-\mathrm{CN})$, is a metal-free semiconductor which is synthesized from low-cost and abundant precursors, ${ }^{19}$ and its photoactivity in the visible range already enabled many interesting applications, i.e., utilizing $\mathrm{g}-\mathrm{CN}$ as a heterogeneous photocatalyst for organic reactions, ${ }^{20}$ photoelectrochemistry, ${ }^{21,22} \mathrm{CO}_{2}$ reduction, ${ }^{23}$ water splitting, ${ }^{24,25}$ or polymer photoinitiator. ${ }^{26,27}$ In the present context, there is only one report on photoinduced $\mathrm{Cu}$-mediated azide-alkyne cycloaddition utilizing $\mathrm{g}$ - $\mathrm{CN}$ as the photocatalyst. $^{28}$ Inspired from the photochemistry of $g-\mathrm{CN}$, here, for the first time, we report on the visible-light-induced dithiol-ene click chemistry between biomass-derivable 4-PEA and 1,2-ethanedithiol (EDT), 2,2-(ethylenedioxy)diethanethiol (EDDT), and 1,4-benzenedimethanethiol (BDT), employing $g-\mathrm{CN}$ as a metal-free photocatalyst (Scheme 1). Furthermore, the synthesis of polyamides from the derived functional dicarboxylic acid was investigated.

Received: September 9, 2019

Revised: October 2, 2019

Published: October 17, 2019 
Scheme 1. Schematic Representation for Dithiol-Ene Clicking between 4-PEA and Different Dithiol Compounds, i.e., EDT, EDDT, and BDT, and Resulting Functional Monomers
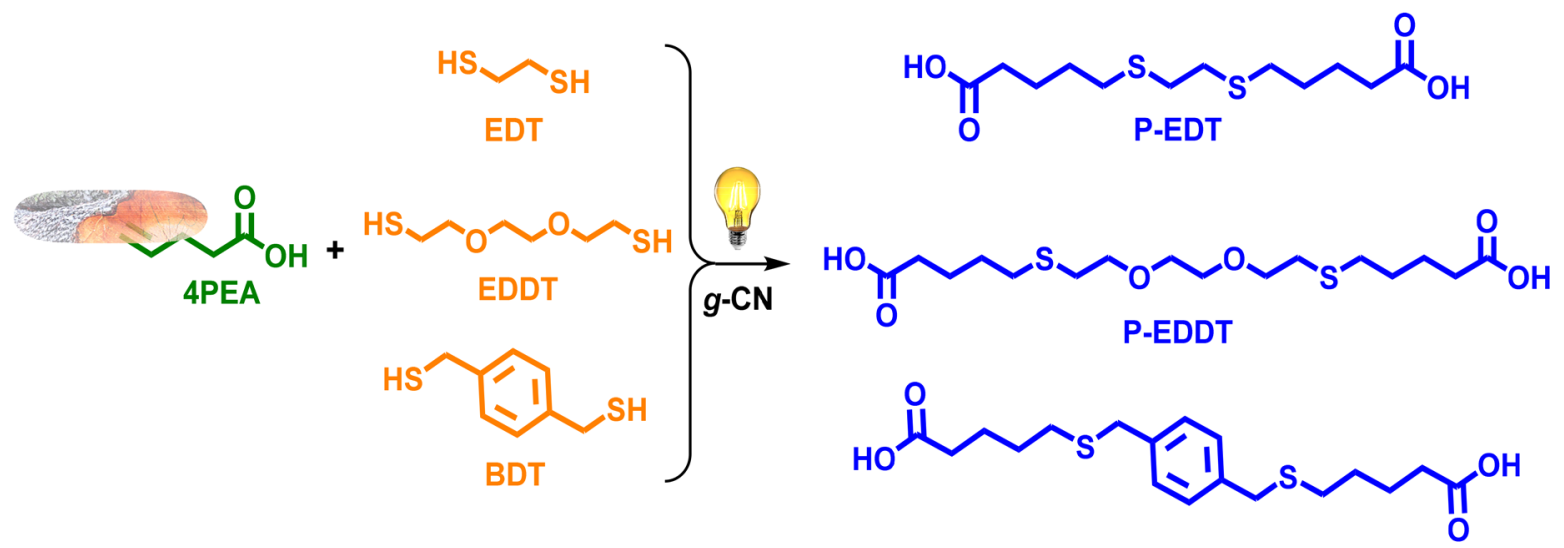

P-BDT

a)

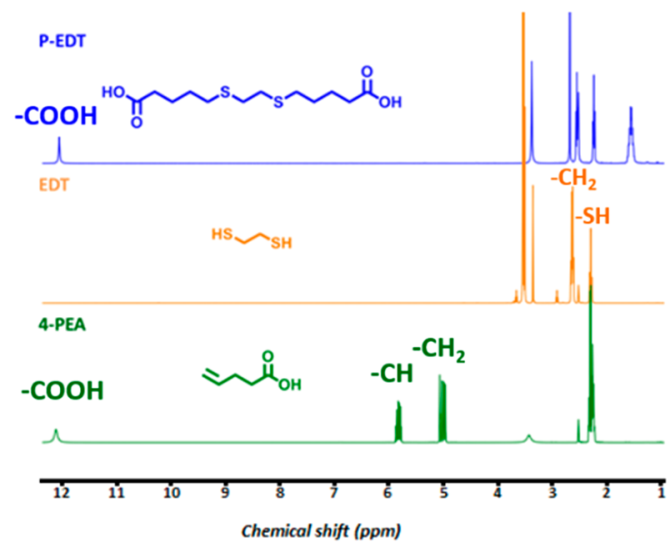

b)

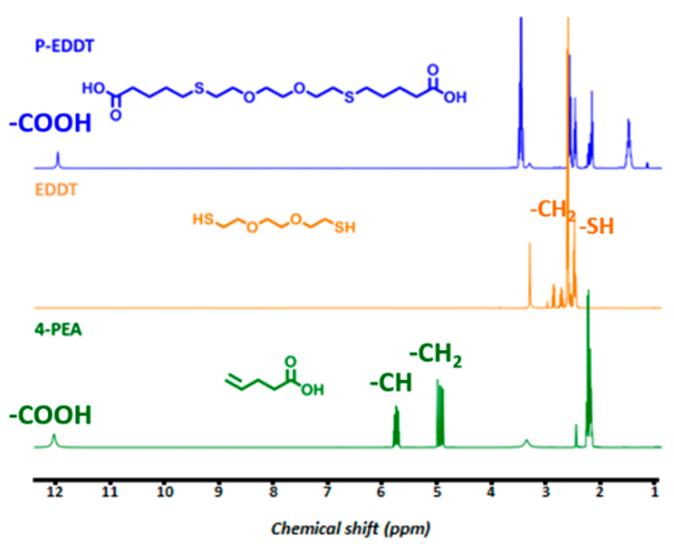

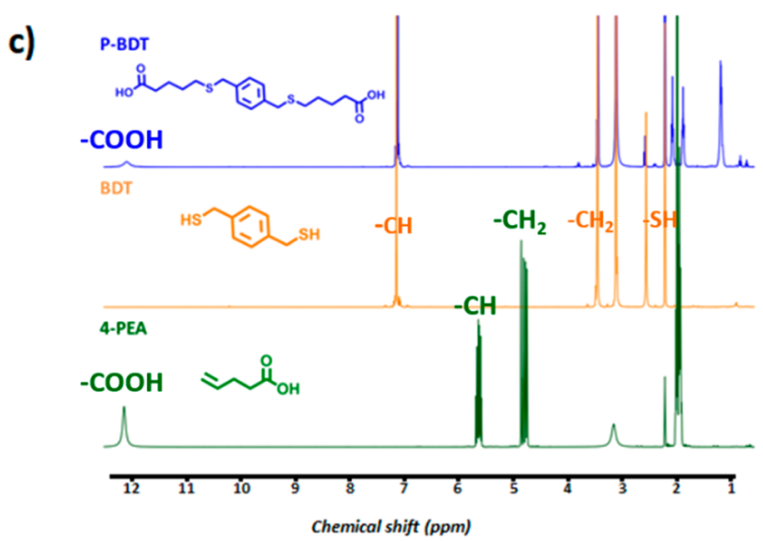

Figure 1. ${ }^{1} \mathrm{H}$ NMR for 4-PEA, EDT, EDDT, and BDT and resulting monomers after dithiol-ene clicking. Reaction conditions: visible light, 5 h; room temperature; 4-PEA/dithiol molar ratio, 2/1; ethanol, $20 \mathrm{~cm}^{3}$; and $g$-CN, $100 \mathrm{mg}$.

\section{RESULTS AND DISCUSSION}

$\mathrm{g}$-CN was synthesized by thermal condensation of a cyanuric acid-melamine complex as reported previously. ${ }^{29,30}$ Solid state $\mathrm{UV}$-vis spectra shows absorbance of $\mathrm{g}$-CN within a visiblelight range, and X-ray diffraction (XRD) presents the typical stacking at $2 \theta$ of $13^{\circ}$ and $27^{\circ}$, which corresponds to intraplanar and interplanar repeat periods (Figure S1).

Dithiol-Ene Clicking Reactions. 4-PEA as being simply available from lignocellulosic biomass was chosen as an exemplary "ene" molecule owing to its functional carboxylic acid group as well as a double bond at the terminal position.
Additionally, dithiol compounds with different main chains (EDT, EDDT, and BDT) were selected to perform dithiolene clicking for the formation of functional dicarboxylic acid monomers which are given the following code in the manuscript: P-EDT, P-EDDT, and P-BDT (Scheme 1, cf. SI for experimental details and reaction conditions). The formation of the dicarboxylic acid monomers (P-EDT, PEDDT, and P-BDT) was confirmed by ${ }^{1} \mathrm{H}$ NMR (Figure 1ac). As can be seen clearly from the ${ }^{1} \mathrm{H}$ NMR spectra, the peaks at chemical shifts of 5.02 and 5.07 ppm for $-\mathrm{CH}_{2}$ and 5.82 $\mathrm{ppm}$ that belong to $-\mathrm{CH}$ groups in 4-PEA are completely 
absent in the formed monomers. Characteristic peaks arising from dithiol compounds are still present in the formed monomers, which indicate the successful clicking reactions. Furthermore, FT-IR spectra for the synthesized monomers show characteristic bands for the $\mathrm{O}-\mathrm{H}$ stretch $(3300-2500$ $\left.\mathrm{cm}^{-1}\right), \mathrm{C}=\mathrm{O}$ stretch $\left(1760-1690 \mathrm{~cm}^{-1}\right), \mathrm{C}-\mathrm{O}$ stretch $\left(1320-1210 \mathrm{~cm}^{-1}\right)$, and $\mathrm{O}-\mathrm{H}$ bend $\left(1440-1395 \mathrm{~cm}^{-1}\right.$ and 950-910 $\mathrm{cm}^{-1}$ ) (Figures S2-S4a). Alternatively, the TGA measurements show the high thermal stability of the synthesized monomers (P-EDT, P-EDDT, and P-BDT), the decomposition of which starts at $573 \mathrm{~K}$ (Figures S2-S4b). These results conclude that 4-PEA can be efficiently clicked with different dithiol compounds in high yields and purities toward rather clean dicarboxylic acids.

In order to understand the dithiol-ene clicking mechanism, a reaction between 4-PEA and EDT was chosen as a reference system. A mixture consisting $\mathrm{g}$-CN (100 mg), 4-PEA (20 g), and EDT $(9.4 \mathrm{~g})$ in ethanol $\left(20 \mathrm{~cm}^{3}\right)$ was stirred for $5 \mathrm{~h}$ in the dark; then, subsequently the ${ }^{1} \mathrm{H}$ NMR of solution was investigated (Figure S5). As expected, the reaction in the dark did not promote any bond formation between dithiol and ene compounds as ${ }^{1} \mathrm{H}$ NMR spectra are similar to the one of the initial mixture (Figure S6a). Therefore, light irradiation is a crucial factor to initiate the reaction. Based on the previous mechanistic studies, the thiol-ene clicking reaction was proven to proceed through a radical pathway. ${ }^{18}$ The visible-lightinduced reaction between 4-PEA and the different dithiols in the presence of $\mathrm{g}$-CN as a photocatalyst follows the radical mechanism as described in Scheme S1. In detail, the visiblelight irradiation generates electrons and holes on $g$-CN. The photoformed electrons react with the thiol groups to develop a thiyl radical anion, where anionic thiyl couples with holes to close the photoredox cycle. The radical thiyls are added to the alkene group of 4-PEA, and proton abstraction propagates the radical pathway. In summary, thiol-ene click chemistry follows a radical pathway, and common thermal initiators like AIBN have a high cost and are single use only. However, $g$-CN offers an alternative solution by being a metal-free and cheap photocatalyst, initiating a photoredox cycle upon visible-light irradiation, and as being a heterogeneous photocatalyst, it promises more than single-time usage.

As a click reaction is visible-light mediated, light on-off cycles were performed in order to investigate the reaction activity in the dark. For this purpose, P-EDT synthesis was chosen as the model reaction for the dithiol-ene click reaction ( $c f$. SI for experimental details). For the ease of investigation, the chemical shift $(12.2 \mathrm{ppm})$ of the ${ }^{1} \mathrm{H}$ NMR for the proton of the carboxylic acid group of 4-PEA was followed as the reference for the reaction progress; it is expected not to undergo any change and/or consumption. Consumed double bond hydrogens of 4-PEA (5.02 and $5.07 \mathrm{ppm}$ ) were noted and related to conversion of 4-PEA $\left({ }^{1} \mathrm{H}\right.$ NMR spectra of light on-off experiments are presented in Figure S6). Within the first $2 \mathrm{~h}$ under visible-light illumination, a $77 \%$ conversion of 4 PEA to P-EDT was observed, and $1 \mathrm{~h}$ of continuous stirring in the dark increased the conversion up to $79 \%$, which confirms the necessity of light illumination for the reaction to proceed efficiently (Figure 2). Irradiation of the mixture via visible light for the next hour (after $4 \mathrm{~h}$ ) enhanced the conversion up to $96 \%$. However, by stirring the mixture subsequently $1 \mathrm{~h}$ in the dark (after $5 \mathrm{~h}$ in total), the conversion of 4-PEA to P-EDT was increased only by another $1 \%$ (conversion after $5 \mathrm{~h}$ is $97 \%$ ). Additionally, XRD of $g$-CN after the photocatalytic

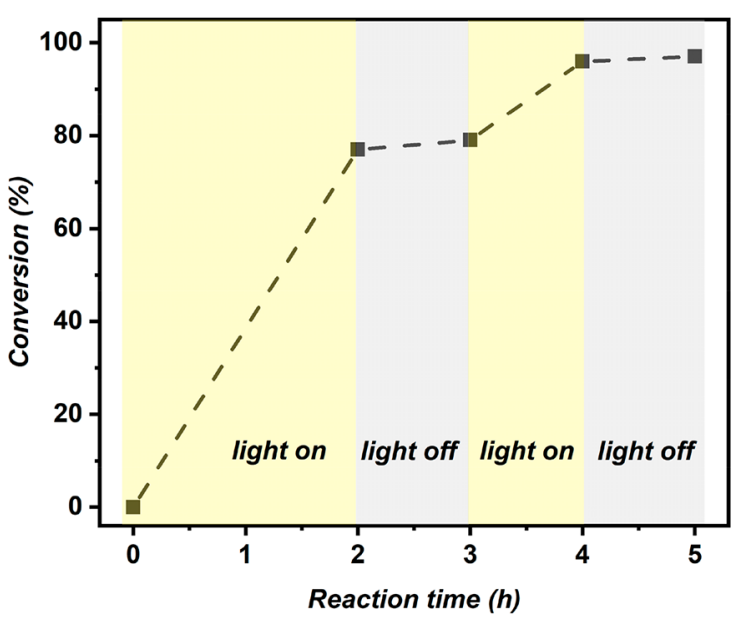

Figure 2. Light on-off experiment results by the means of total conversion of 4-PEA to P-EDT synthesis. Reaction conditions: $\mathrm{m}_{4-\mathrm{PEA}}$, $1.0 \mathrm{~g} ; \mathrm{m}_{\mathrm{EDT}}, 0.47 \mathrm{~g} ; \mathrm{m}_{\mathrm{g}-\mathrm{CN}}, 0.01 \mathrm{~g} ; \mathrm{T}, 298 \mathrm{~K}$, and under visible light irradiation.

reaction presents a similar profile as the fresh one, which underlines the stability of $\mathrm{g}$-CN after $5 \mathrm{~h}$ of photocatalytic reaction (Figure S7), as otherwise structural changes are observable via XRD. ${ }^{31}$ XPS was conducted for freshly synthesized (Figure S8a) and isolated $g$-CN after the photocatalytic reaction (Figure S8b), which shows similar C $1 \mathrm{~s}$ and $\mathrm{N} 1 \mathrm{~s}$ spectra and similar as reported in the literature, ${ }^{32}$ as another indication for the stability of the catalyst.

The effect of photocatalyst amount was investigated via taking P-EDDT synthesis as a model reaction. In this regard, the amounts of 4-PEA and EDDT were kept constant, while only the $g$-CN amount was tripled, and the mixture was irradiated for $3 \mathrm{~h}$. Both ${ }^{1} \mathrm{H}$ NMR spectra presented similar peaks with high conversion values, where the conversion difference between the two systems was less than 5\% (Figure S9). These results indicate that the photoactivity of $g-\mathrm{CN}$ saturates and relies on little catalyst, as even $0.15 \mathrm{wt} \% \mathrm{~g}-\mathrm{CN}$ is enough to catalyze the visible-light-induced dithiol-ene clicking reaction with high efficiencies in short times. This is expected for such very active photoabsorbers.

The structure of P-EDDT is slightly similar to poloxamer compounds, which are ABA-type block copolymer-based surfactants with significant industrial interest. ${ }^{33}$ Indeed, not only the monomer but also the viscous polymer liquid PEDDT can be utilized directly as a surfactant to form oil in water emulsions (Figure S10).

Polyamides from Synthesized Functionalized Dicarboxylic Acids. The dicarboxylic acid functional monomers were then utilized for polymer synthesis. For this purpose, polyamide synthesis from P-EDT, P-EDDT, and P-BDT monomers was investigated (see SI for detailed polyamides synthesis procedure and Figure S11 for digital images of polymers). Polymerization via condensation reactions using azeotropic distillation in a Dean-Stark setup with toluene at $403 \mathrm{~K}$ for $16 \mathrm{~h}$ without any catalyst was performed. The choice of diamine is varied to give different main chain structures with linear, amphiphilic ethylenedioxy, and aromatic units. The synthesized polymers are annotated as poly(P-EDT), poly(PEDDT), and poly(P-BDT) in this manuscript. For the monomer (P-EDT), polymerization with 1,6-hexamethylenediamine yielded a polymer with an average molecular weight $\left(M_{\mathrm{w}}\right)$ of $9650 \mathrm{~g} \mathrm{~mol}^{-1}$ and molecular dispersities $(\boxplus)$ of a 1.90 
a)

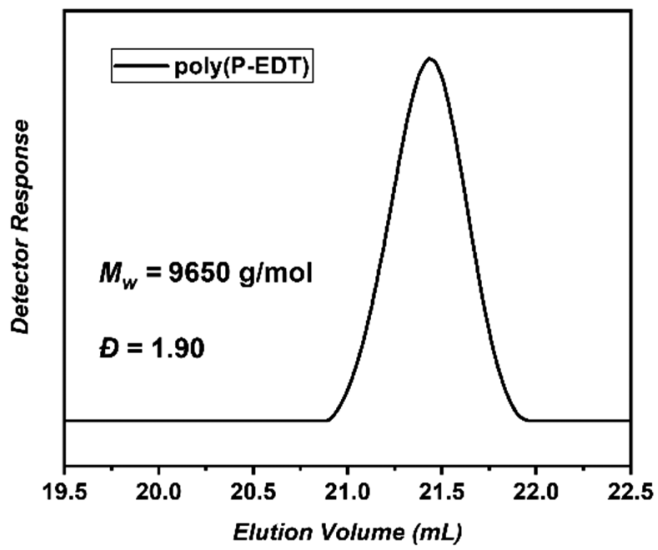

b)

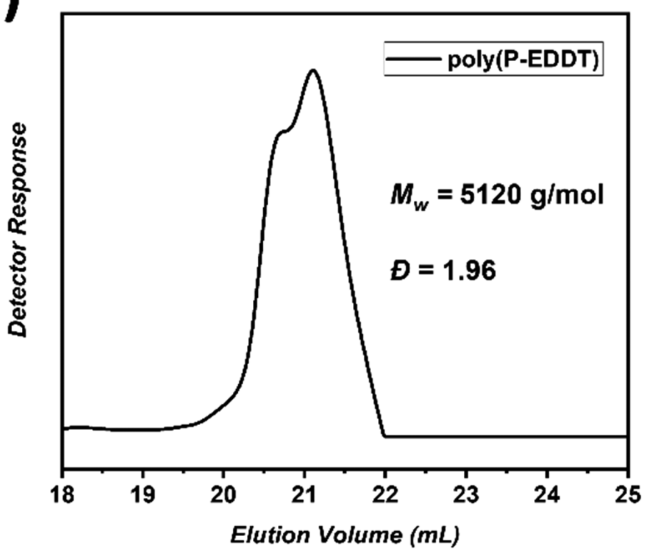

Figure 3. SEC traces of (a) poly(P-EDT) was synthesized using P-EDT (0.01 mol) and 1,6-hexamethylenediamine (0.01 mol). (b) Poly(P-EDDT) was synthesized using P-EDDT $(0.01 \mathrm{~mol})$ and Jeffamine T-403 $(0.01 \mathrm{~mol})$. The samples were measured via NMP as solvent relative to polystyrene to calibrate the column.

derived size exclusion chromatography (Figure 3a). Additionally, TGA of poly(P-EDT) showed thermal stability up to 573 $\mathrm{K}$, where significant mass loss starts around $603 \mathrm{~K}$ with less than $3 \%$ remaining at $773 \mathrm{~K}$ (Figure S12). Moreover, TGA showed that the thermal stability has been improved (373 K) upon polymerization of P-EDT (Figures S2a and S12). Differential scanning calorimetry (DSC) for the synthesized polymer, i.e., poly(P-EDT), showed a double meeting peaks which correspond to the presence of multiple phase and different crystallinity (Figure S13a). Also, the presence of the impurities upon removing the solvent can be another reason for these peaks. Thus, the glass transition temperature $\left(T_{\mathrm{g}}\right)$ cannot be identified. Furthermore, FT-IR spectra of poly(PEDT) presents a carboxyl peak around $1650 \mathrm{~cm}^{-1}$ and retains the main chain vibrations compared to P-EDT (Figure S14a). However, new peaks arising from the amide bond formation were observed (at $3300 \mathrm{~cm}^{-1}$ ), which confirms the polyamide formation. XRD investigation was performed for poly(P-EDT) as polyamides, in general, possess partial crystallinity. In addition to the amorphous side, poly(P-EDT) also demonstrates crystalline domains, even though less pronounced, indicating a semicrystalline polymer character (Figure S15a). Partial crystallinity is a mandatory requirement for polyamides to be technologically useful, extending the application range up to slightly below the melting point.

Polymerization of P-EDDT was performed with Jeffamine $\mathrm{T}-403$ in order to introduce ethylenedioxy-based repeating units in the synthesized polyamide. The resulting polymer was a highly viscous brown liquid (Figure S11), where SEC has presented a polymer with $M_{\mathrm{w}}$ of $5120 \mathrm{~g} \mathrm{~mol}^{-1}$ and $Ð$ of 1.96 , $v i z$ in Figure $3 \mathrm{~b}$. The poly(P-EDDT) demonstrated high thermal stability which is comparable to poly(P-EDT), up to $573 \mathrm{~K}$ and less than $3 \mathrm{wt} \%$ remaining at $773 \mathrm{~K}$ (Figure S12). Since poly(P-EDDT) is a viscous liquid, it comes with a low $T_{\mathrm{g}}$ value (around $309 \mathrm{~K}$ ) confirmed by DSC (Figure S13b). Additionally, FT-IR spectra of poly(P-EDDT) showed a typical carboxyl absorbance band at $1650 \mathrm{~cm}^{-1}$ and retained the main chain vibrations, especially etheric $\mathrm{C}-\mathrm{O}$ stretching at $1050 \mathrm{~cm}^{-1}$, compared to P-EDDT (Figure S14b). However, new peaks arising from amide bond formation were observed (mainly at a wavenumber of $3300 \mathrm{~cm}^{-1}$ ). The XRD profile of liquid poly(P-EDDT) reflects a locally amorphous structure (Figure S15b). In order to explore the flow behavior of poly(P-
EDDT), viscosity was investigated via rheology (Figure S16a). The poly(P-EDDT) possessed a dilatant (shear thickening) behavior, in which the amorphous polymer chains even undergo flocculation upon increased shear (Figure S16b). This is a typical for amphiphilic block copolymers with rheoordering. On the other hand, increasing the temperature resulted in a dramatic decrease in the polymer viscosity.

Polymerization of P-BDT was also performed with phenylene diamine to present an aramide-type polymer. However, the investigation of molecular weight and polydispersity of the synthesized polymer, i.e., poly(P-BDT), via SEC was not possible due to the insolubility of the crystalline domains in any solvent, e.g., $\mathrm{N}$-methyl-2-pyrrolidone (NMP), tetrahydrofuran (THF), and water, in which SEC can be measured. Nevertheless, poly(P-BDT) presented a lower thermal stability with respect to poly(P-EDT) and poly(P-EDDT), despite more solid content retained above $773 \mathrm{~K}$ (Figure S12). Similarly to poly(P-EDT), the synthesized polymer showed double meeting peaks that can correspond to the multiple phases with different crystallinity. In this context, the $T_{\mathrm{g}}$ value cannot be identified (Figure S13c). Also, FT-IR spectra of poly(P-BDT) showed a carboxyl vibration band around 1650 $\mathrm{cm}^{-1}$, and the main chain vibrations remain preserved, especially benzylic stretchings at 750 and $1400-1600 \mathrm{~cm}^{-1}$, compared to P-BDT (Figure S14c). Amide bond formation was confirmed also via FT-IR by the appearance of the absorbance band mainly at $3300 \mathrm{~cm}^{-1}$. The XRD diffraction pattern of poly(P-BDT) showed a semicrystalline structure (Figure S15c) due to strong $\pi-\pi$ aromatic stackings and inhibition of amorphous polymer domains.

\section{CONCLUSION}

The future biomass platform molecule 4-pentenoic acid was used in a green, sustainable, and cost-efficient synthesis, based on a visible-light-induced dithiol-ene click reaction using metal-free $g$-CN as a photocatalyst. The utilization of three different dithiol compounds for the synthesis of functional dicarboxylic acids resulted in the formation of functional dicarboxylic acid monomers in complete yield and high purity and illustrated the versatility of this reaction. In the presence of $g-\mathrm{CN}$, light irradiation is crucial to accelerate the clicking reaction between 4-pentenoic acid and dithiol compounds via radical mechanism. Polycondensation with structurally similar 
diamine compounds was applied to synthesize polyamides. The linear and aromatic compounds exhibited a high glass transition temperature $(373 \mathrm{~K})$. Alternatively, the monomercontaining ethylenedioxy unit can act as a surfactant to stabilize oil/water emulsions. This study is envisaging the way for broadening the utilization of $g$ - $\mathrm{CN}$ as a photocatalyst in sustainable processes.

\section{ASSOCIATED CONTENT}

\section{S Supporting Information}

The Supporting Information is available free of charge on the ACS Publications website at DOI: 10.1021/acssuschemeng.9b05351.

Chemicals, synthetic procedures, characterization, $g-\mathrm{CN}$ characterization, TGA, FT-IR of synthesized monomers and polymers, ${ }^{1} \mathrm{H}$ NMR of kinetic studies, XRD and DSC of polymers, and rheology results of poly(PEDDT). (PDF)

\section{AUTHOR INFORMATION}

\section{Corresponding Author}

*E-mail: majd.al-naji@mpikg.mpg.de. Tel (+49) 331567 9569. Fax (+49) 3315679502.

\section{ORCID}

Markus Antonietti: 0000-0002-8395-7558

Majd Al-Naji: 0000-0001-5024-0449

\section{Author Contributions}

${ }^{\ddagger}$ Baris Kumru and Jose Mendoza Mesa contributed equally. The manuscript was written through contributions of all authors. All authors have given approval to the final version of the manuscript.

\section{Notes}

The authors declare no competing financial interest.

\section{ACKNOWLEDGMENTS}

The authors thank the Max Planck Society for funding. Antje Volkel is greatly acknowledged for TGA and DSC measurements, and we thank Jianrui Zhang for microscopy imaging. We are thankful for Marlies Grawert for SEC measurements and the electrical workshop at the Max Planck Institute of Colloids and Interfaces for helping with visible-light (LED) setups. Dr. Kerstin Blank is acknowledged for rheometer access, and the technical support by Irina Shekova is greatly acknowledged. Jose Mendoza Mesa is grateful to "Gobernación del Tolima" and Colfuturo for a research scholarship granted through Convocatoria 727-2017 of Colciencias at the Max Planck Institute of Colloids and Interfaces.

\section{REFERENCES}

(1) Ten, E.; Vermerris, W. Recent developments in polymers derived from industrial lignin. J. Appl. Polym. Sci. 2015, 132 (24), 42069.

(2) Agbor, V. B.; Cicek, N.; Sparling, R.; Berlin, A.; Levin, D. B. Biomass pretreatment: Fundamentals toward application. Biotechnol. Adv. 2011, 29, 675-685.

(3) Al-Naji, M.; Puertolas, B.; Kumru, B.; Cruz, D.; Baumel, M.; Schmidt, B. V. K. J.; Tarakina, N. V.; Perez-Ramirez, J. Sustainable Continuous Flow Valorization of $\gamma$-Valerolactone with Trioxane to $\alpha$ Methylene- $\gamma$-Valerolactone over Basic Beta Zeolite. ChemSusChem 2019, 12 (12), 2628-2636.

(4) Al-Naji, M.; Yepez, A.; Balu, A. M.; Romero, A. A.; Chen, Z.; Wilde, N.; Li, H.; Shih, K.; Glaser, R.; Luque, R. Insights into the selective hydrogenation of levulinic acid to-valerolactone using supported mono- and bimetallic catalysts. J. Mol. Catal. A: Chem. 2016, 417, 145-152.

(5) Bond, J. Q.; Alonso, D. M.; West, R. M.; Dumesic, J. A. $\gamma$ Valerolactone Ring-Opening and Decarboxylation over $\mathrm{SiO}_{2} / \mathrm{Al}_{2} \mathrm{O}_{3}$ in the Presence of Water. Langmuir 2010, 26 (21), 16291-16298.

(6) Bond, J. Q.; Jungong, C. S.; Chatzidimitriou, A. Microkinetic analysis of ring opening and decarboxylation of cvalerolactone over silica alumina. J. Catal. 2016, 344, 640-656.

(7) Low, C. H.; Nobbs, J. D.; van Meurs, M.; Stubbs, L. P.; Drent, E.; Aitipamula, S.; Pung, M. H. L. Palladium Complexes with Bulky Diphosphine Ligands as Highly Selective Catalysts for the Synthesis of (Bio-) Adipic Acid from Pentenoic Acid Mixtures. Organometallics 2015, 34, 4281-4292.

(8) Zhao, L.; Pudasaini, B.; Genest, A.; Nobbs, J. D.; Low, C. H.; Stubbs, L. P.; van Meurs, M.; Rosch, N. Palladium-Catalyzed Hydroxycarbonylation of Pentenoic Acids. Computational and Experimental Studies on the Catalytic Selectivity. ACS Catal. 2017, 7, 7070-7080

(9) Nobbs, J. D.; Zainal, N. Z. B.; Tan, J.; Drent, E.; Stubbs, L. P.; Li, C.; Lim, S. C. Y.; Kumbang, D. G. A.; van Meurs, M. Bio-based Pentenoic Acids as Intermediates to Higher Value-Added Mono- and Dicarboxylic Acids. ChemistrySelect 2016, 1, 539-544.

(10) Palkovits, R. Pentenoic Acid Pathways for Cellulosic Biofuels. Angew. Chem., Int. Ed. 2010, 49, 4336-4338.

(11) Rorrer, N. A.; Vardon, D. R.; Dorgan, J. R.; Gjersing, E. J.; Beckham, G. T. Biomass-derived monomers for performancedifferentiated fiber reinforced polymer composites. Green Chem. 2017, 19, 2812-2825.

(12) Lowe, A. B. Thiol-ene "click" reactions and recent applications in polymer and materials synthesis: a first update Polym. Polym. Chem. 2014, 5, 4820-4870.

(13) Kempe, K.; Krieg, A.; Becer, C. R.; Schubert, U. S. Clicking” on/with polymers: a rapidly expanding field for the straightforward preparation of novel macromolecular architectures. Chem. Soc. Rev. 2012, 41, 176-191.

(14) Isikgor, F. H.; Becer, C. R. Lignocellulosic biomass: a sustainable platform for the production of bio-based chemicals and polymers. Polym. Chem. 2015, 6, 4497-4559.

(15) Dondoni, A.; Marra, A. Recent applications of thiol-ene coupling as a click process for glycoconjugation. Chem. Soc. Rev. 2012, $41,573-586$.

(16) Bhat, V. T.; Duspara, P. A.; Seo, S.; Abu Bakar, N. S. B.; Greaney, M. F. Visible light promoted thiol-ene reactions using titanium dioxide. Chem. Commun. 2015, 51, 4383-4385.

(17) Tyson, E. L.; Niemeyer, Z. L.; Yoon, T. P. Redox Mediators in Visible Light Photocatalysis: Photocatalytic Radical Thiol-Ene Additions. J. Org. Chem. 2014, 79 (3), 1427-1436.

(18) Li, Y.; Cai, J.; Hao, M.; Li, Z. Visible light initiated hydrothiolation of alkenes and alkynes over $\mathrm{ZnIn}_{2} \mathrm{~S}_{4}$. Green Chem. 2019, 21, 2345-2351.

(19) Liu, J.; Wang, H.; Antonietti, M. Graphitic carbon nitride "reloaded": emerging applications beyond (photo)catalysis. Chem. Soc. Rev. 2016, 45 (8), 2308-26.

(20) Datta, K. K.; Reddy, B. V.; Ariga, K.; Vinu, A. Gold nanoparticles embedded in a mesoporous carbon nitride stabilizer for highly efficient three-component coupling reaction. Angew. Chem., Int. Ed. 2010, 49 (34), 5961-5.

(21) Kumru, B.; Barrio, J.; Zhang, J.; Antonietti, M.; Shalom, M.; Schmidt, B. V. K. J. Robust Carbon Nitride-Based Thermoset Coatings for Surface Modification and Photochemistry. ACS Appl. Mater. Interfaces 2019, 11 (9), 9462-9469.

(22) Xu, J.; Herraiz-Cardona, I.; Yang, X.; Gimenez, S.; Antonietti, M.; Shalom, M. The Complex Role of Carbon Nitride as a Sensitizer in Photoelectrochemical Cells. Adv. Opt. Mater. 2015, 3 (8), 10521058.

(23) Lin, J.; Pan, Z.; Wang, X. Photochemical Reduction of CO2by Graphitic Carbon Nitride Polymers. ACS Sustainable Chem. Eng. 2014, 2 (3), 353-358. 
(24) Schwinghammer, K.; Tuffy, B.; Mesch, M. B.; Wirnhier, E.; Martineau, C.; Taulelle, F.; Schnick, W.; Senker, J.; Lotsch, B. V. Triazine-based carbon nitrides for visible-light-driven hydrogen evolution. Angew. Chem., Int. Ed. 2013, 52 (9), 2435-9.

(25) Thaweesak, S.; Wang, S.; Lyu, M.; Xiao, M.; Peerakiatkhajohn, P.; Wang, L. Boron-doped graphitic carbon nitride nanosheets for enhanced visible light photocatalytic water splitting. Dalton Trans 2017, 46, 10714-10720.

(26) Kumru, B.; Cruz, D.; Heil, T.; Schmidt, B. V. K. J.; Antonietti, M. Electrostatic Stabilization of Carbon Nitride Colloids in Organic Solvents Enables Stable Dispersions and Transparent Homogeneous CN-Films for Optoelectronics. J. Am. Chem. Soc. 2018, 140 (50), 17532-17537.

(27) Cao, Q.; Kumru, B.; Antonietti, M.; Schmidt, B. V. K. J. Grafting Polymers onto Carbon Nitride via Visible-Light-Induced Photofunctionalization. Macromolecules 2019, 52 (13), 4989-4996.

(28) Dadashi-Silab, S.; Kiskan, B.; Antonietti, M.; Yagci, Y. Mesoporous graphitic carbon nitride as a heterogeneous catalyst for photoinduced copper(I)-catalyzed azide-alkyne cycloaddition. RSC Adv. 2014, 4, 52170-52173.

(29) Shalom, M.; Inal, S.; Fettkenhauer, C.; Neher, D.; Antonietti, M. Improving carbon nitride photocatalysis by supramolecular preorganization of monomers. J. Am. Chem. Soc. 2013, 135 (19), $7118-21$.

(30) Kumru, B.; Molinari, V.; Hilgart, M.; Rummel, F.; Schaeffler, M.; Schmidt, B. V. K. J. Polymer Grafted Graphitic Carbon Nitride as Precursors for Reinforced Lubricant Hydrogels. Polym. Chem. 2019, 10, 3647-3656.

(31) Kumru, B.; Molinari, V.; Dunnebacke, R.; Blank, K. G.; Schmidt, B. V. K. J. Extremely Compressible Hydrogel via Incorporation of Modified Graphitic Carbon Nitride. Macromol. Rapid Commun. 2018, 40 (4), 1800712.

(32) Gu, S.; Xie, J.; Li, M. C. Hierarchically porous graphitic carbon nitride: large-scale facile synthesis and its application toward photocatalytic dye degradation. RSC Adv. 2014, 4, 59436-59439.

(33) Alexandridis, P.; Hatton, T. A. Poly(ethylene oxide)-poly(propylene oxide)-poly(ethylene oxide) block copolymer surfactants in aqueous solutions and at interfaces: thermodynamics, structure, dynamics, and modeling. Colloids Surf., A 1995, 96 (1), 1-46. 